\title{
Téoros
}

Revue de recherche en tourisme

\section{Et au milieu coule une rivière}

Patrimoine industriel, tourisme et développement durable

\section{Mathieu Dormaels}

Volume 30, numéro 1, 2011

URI : https://id.erudit.org/iderudit/1012116ar

DOI : https://doi.org/10.7202/1012116ar

Aller au sommaire du numéro

Éditeur(s)

Université du Québec à Montréal

ISSN

0712-8657 (imprimé)

1923-2705 (numérique)

Découvrir la revue

Citer cet article

Dormaels, M. (2011). Et au milieu coule une rivière : patrimoine industriel, tourisme et développement durable. Téoros, 30(1), 139-142.

https://doi.org/10.7202/1012116ar d'utilisation que vous pouvez consulter en ligne.

https://apropos.erudit.org/fr/usagers/politique-dutilisation/ 


\title{
Et au milieu coule une rivière Patrimoine industriel, tourisme et développement durable
}

\author{
Mathieu DORMAELS \\ Chercheur associé \\ Université du Québec à Montréal (ESG-UQAM) \\ mat_dormaels@yahoo.com
}

Dans bien des cas, le patrimoine tend à nous rappeler le rôle particulier des ressources naturelles dans le développement des communautés humaines. Implantées souvent au bord d'un cours d'eau, nos villes gardent les traces de ce choix originel dans leur forme - comme les rives du fleuve à Montréal ou Québec - ou dans leur nom. La révolution industrielle et le développement qu'elle supposa à la fin du XIX ${ }^{\mathrm{e}}$ siècle et au début du $\mathrm{XX}^{\mathrm{e}}$ siècle en Amérique du Nord est une période qui a laissé un grand nombre de ces témoins architecturaux et urbains. On connaît au Québec plusieurs villes qui se sont développées autour d'industries qui avaient besoin du pouvoir de l'eau. Certaines de ces usines fonctionnent encore, comme les installations de Rio Tinto Alcan au Saguenay, et notamment celle d'Arvida. Par contre, d'autres ont dû fermer, laissant la ville abandonnée, comme à Val-Jalbert, ou impliquant de nouveaux types d'activités économiques. Les espaces laissés vides doivent alors être convertis pour de nouveaux usages et les fonctions touristique et culturelle ont été, ces dernières années, des options largement choisies entre toutes, comme à Shawinigan avec la Cité de l'énergie, par exemple ${ }^{1}$. Le sujet n'est pas nouveau, mais des tendances s'y affirment qui apparaissent intéressantes à soulever. En effet, ces transformations semblent de plus en plus mettre en avant des valeurs qui sont relativement contradictoires avec l'histoire industrielle que portent ces lieux. On observe que le souci de sauvegarde s'accompagne davantage de préoccupations sociales d'amélioration des conditions de vie et de préoccupations environnementales de récupération des espaces naturels. De plus en plus, les opérations de restauration, de réhabilitation et de reconversion de ces édifices doivent répondre à des standards nouveaux de développement durable et de responsabilité sociale. Bien que cette contradiction avec l'activité industrielle passée ne soit évidemment qu'apparente, elle justifie au minimum que l'on s'interroge sur ce type de réaffectation, ce que l'on se propose de faire ici à travers deux exemples.

De l'autre côté de la frontière sud du Québec, on observe des situations comparables, mais avec un nombre sans doute plus important de sites. Cette région, autrefois intégrée à la Nouvelle-Angleterre, a connu elle aussi un développement très important dans la seconde moitié du $\mathrm{XIX}^{\mathrm{e}}$ siècle et au début du $\mathrm{XX}^{\mathrm{e}}$ siècle. De nombreuses manufactures se sont implantées le long des principaux cours d'eau, sous le nom générique de «Mills» (Moulins), puisqu'elles profitaient de la force hydromotrice pour leur fonctionnement, mais aussi de leur navigabilité pour le transport des marchandises. Le textile a été la plus importante de ces industries, et on en reconnaît les traces, notamment à Lowell (Massachusetts) où de grands ensembles ont été préservés et font partie du Lowell National Historical Park. Cependant, il existait aussi beaucoup d'autres industries, de tailles plus modestes, comme l'usine d'armes Robbins and Lawrence à Windsor, l'usine de papier de Bellows Falls ou la Estey Organ Company, une fabrique de Reed Organs (une sorte d'harmonium) à Battleboro. Ces trois villes forment, avec d'autres, un ensemble de sites à proximité de la rivière Connecticut et regroupés dans un Byway (le nom complet du programme étant The National Scenic Byways Program), c'est-à-dire une route patrimoniale reconnue par le ministère des Transports (U.S. Department of transportation). Dans beaucoup de cas comme celui de Lowell, plus proche de Boston et de ses centres universitaires, les nouvelles technologies, la recherche et l'éducation ont permis de dynamiser à nouveau ces villes et de conserver le patrimoine bâti en lui affectant de nouveaux usages. En revanche, dans les communautés plus éloignées du Vermont ou du New Hampshire, ces solutions n'existent pas.

Les villes de ces États ont donc souvent opté pour le tourisme et la culture, mais cette solution n'est évidemment ni magique ni facile. Dans certains cas, lorsque l'on dispose d'une histoire particulièrement dense et d'artefacts nombreux, on peut envisager l'implantation d'un musée, comme c'est le cas à Windsor (Vermont). Le American Precision Museum, installé dans l'ancienne usine d'armes, propose un regard sur l'évolution technologique qui a permis la production standardisée et de masse à travers l'histoire de ce lieu d'innovation et de 


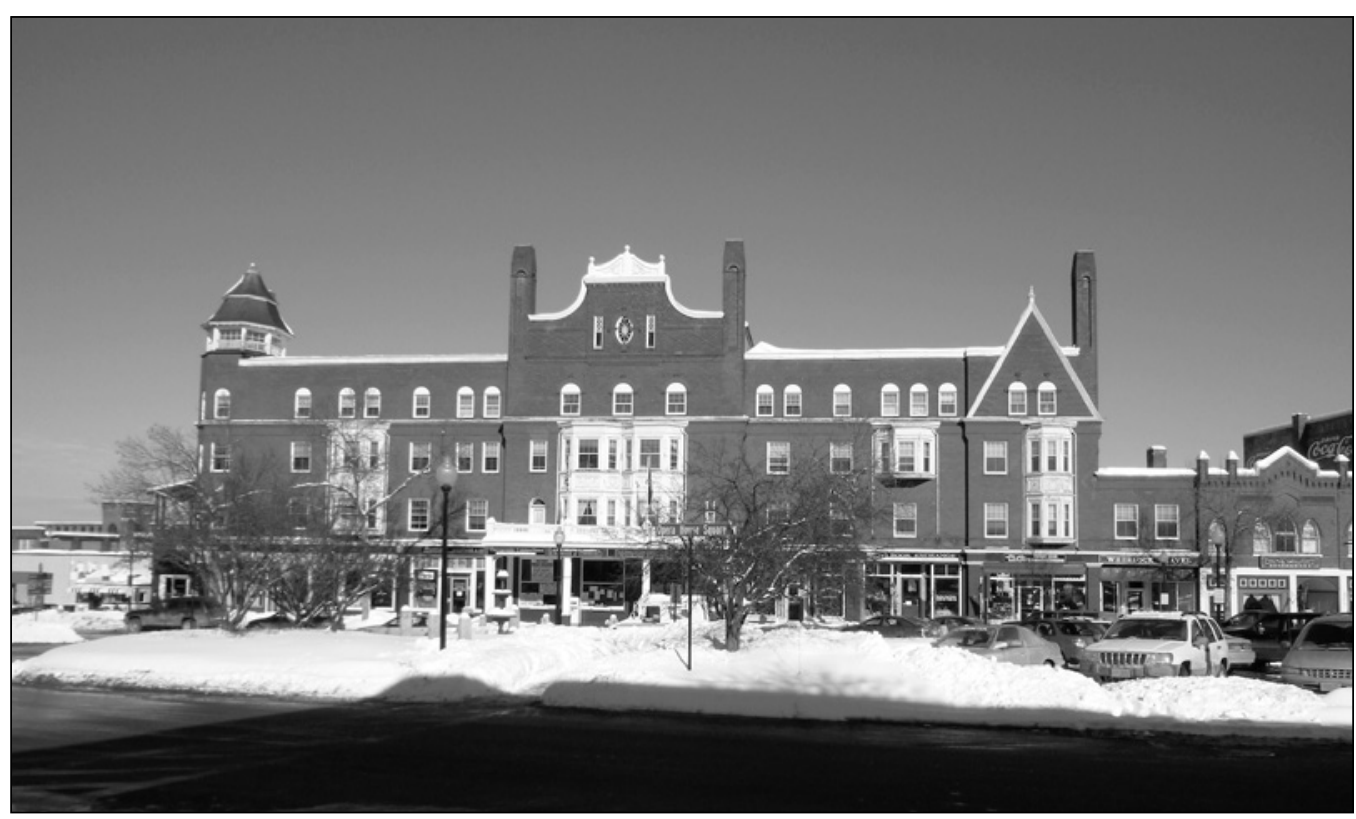

ILLUSTRATION 1 : Moody Building, Claremont, New Hampshire (photo : Mathieu Dormaels). production. Dans le cas de White River Junction (Vermont), c'est l'histoire du transport qui est interprétée dans l'ancienne gare de chemin de fer. Cette ville, construite au confluent de la White River et de la rivière Connecticut, doit son importance à la convergence de cinq lignes de voies ferrées construites entre 1847 et 1863, et qui en fit un centre important pour le transport de marchandises produites dans les fermes et les manufactures de la région. Toutefois, dans d'autres cas, il semble peser un déséquilibre entre l'importance du bâti et la taille de la ville. C'est ce qu'on pourrait penser du cas de Claremont (New Hampshire), elle aussi partie intégrante de l'ensemble des sites de la rivière Connecticut, bien que située sur la Sugar River, près du confluent. En effet, le centre de cette ville d'environ 13000 habitants est massivement occupé par les imposants édifices industriels en brique qui servaient à la production textile. En fait, c'est une grande partie du paysage urbain du centre-ville qui a été construite entre 1880 et 1905, alors que la ville connaissait un boom démographique de 4000 habitants vers 1850 à 7000 au début du XX ${ }^{\mathrm{e}}$ siècle (Edmond, 1991 : 13). Les principaux bâtiments non industriels encore visibles aujourd'hui datent de cette période, comme le Moody Building, anciennement Hotel Claremont (voir illustration 1) de 1892, le Claremont City Hall and Opera House (deux édifices reliés aujourd'hui par une extension moderne) de 1897, et la bibliothèque Fiske Free de 1903. À côté de ceux-ci, au bord du cours d'eau, on trouve les anciennes fabriques dont plus aucune n'a conservé sa vocation industrielle (voir illustration 2 ).

Si la ville a subi le même déclin que les autres avec la crise économique récente, on voit que, sur le fond, l'activité manufacturière a considérablement baissé en 10 ans (659 emplois en 2009 contre 1802 en 1999, selon les chiffres des services de l'État du New Hampshire), laissant place aux activités de service. C'est donc logiquement que l'on retrouve ce type d'activités dans les anciens bâtiments avec notamment l'aménagement d'espaces de bureaux, et d'un hôtel haut de gamme et de son restaurant. Par contre, de chaque côté, les imposantes constructions restent vides, l'une interrompue dans sa conversion en appartements de luxe, l'autre simplement fermée et sans activité. C'est aussi vers le tourisme que se tourne la ville : tourisme de détente, en été comme en hiver, avec les ressources naturelles de la région pour la randonnée, le ski et les activités nautiques, et tourisme d'affaires avec les espaces de l'hôtel. Cependant, il doit s'agir d'un développement touristique qui profite à l'ensemble de la communauté, celle-ci devant démontrer son implication et son dynamisme pour recevoir la reconnaissance qui enrichira son offre touristique. C'est bien ainsi que les organismes gouvernementaux soutiennent ces initiatives, en reconnaissant la valeur patrimoniale de la rivière et de ses abords, mais aussi la qualité de la préservation de l'environnement et de l'implication des citoyens. Ce sont là les conditions essentielles qui furent retenues dans la désignation des 14 American Heritage Rivers par le président Clinton en juillet 1998, et dont fait partie la rivière Connecticut et ses affluents. C'est aussi la «qualité de la vie», en plus de la beauté exceptionnelle, qui est reconnue par la désignation du ministère des Transports à travers le programme National Scenic Byways. Toutefois, il apparaît clairement que le seul tourisme n'est pas suffisant pour justifier et financer le maintien de tels bâtiments, et si la ville cherche à améliorer la qualité de vie de ses habitants, c'est aussi pour attirer de nouvelles entreprises. C'est bien sûr l'objectif de la campagne de communication des autorités locales baptisée The Claremont advantage, mais c'est aussi une des raisons pour lesquelles la ville procède à l'aménagement des rives et de sentiers de promenades. Justement, et c'est ce qui reste dans une certaine mesure contradictoire, l'une des tâches les plus importantes a été de nettoyer les traces polluantes de l'activité industrielle passée sur l'environnement.

Le cas est différent, mais les motivations identiques, pour la ville de Burlington (Vermont). Située sur la rive est du 


\section{ILLUSTRATION 2 : \\ Anciens bâtiments industriels au bord de la Sugar River, Claremont, New Hampshire (photo : Mathieu Dormaels).}

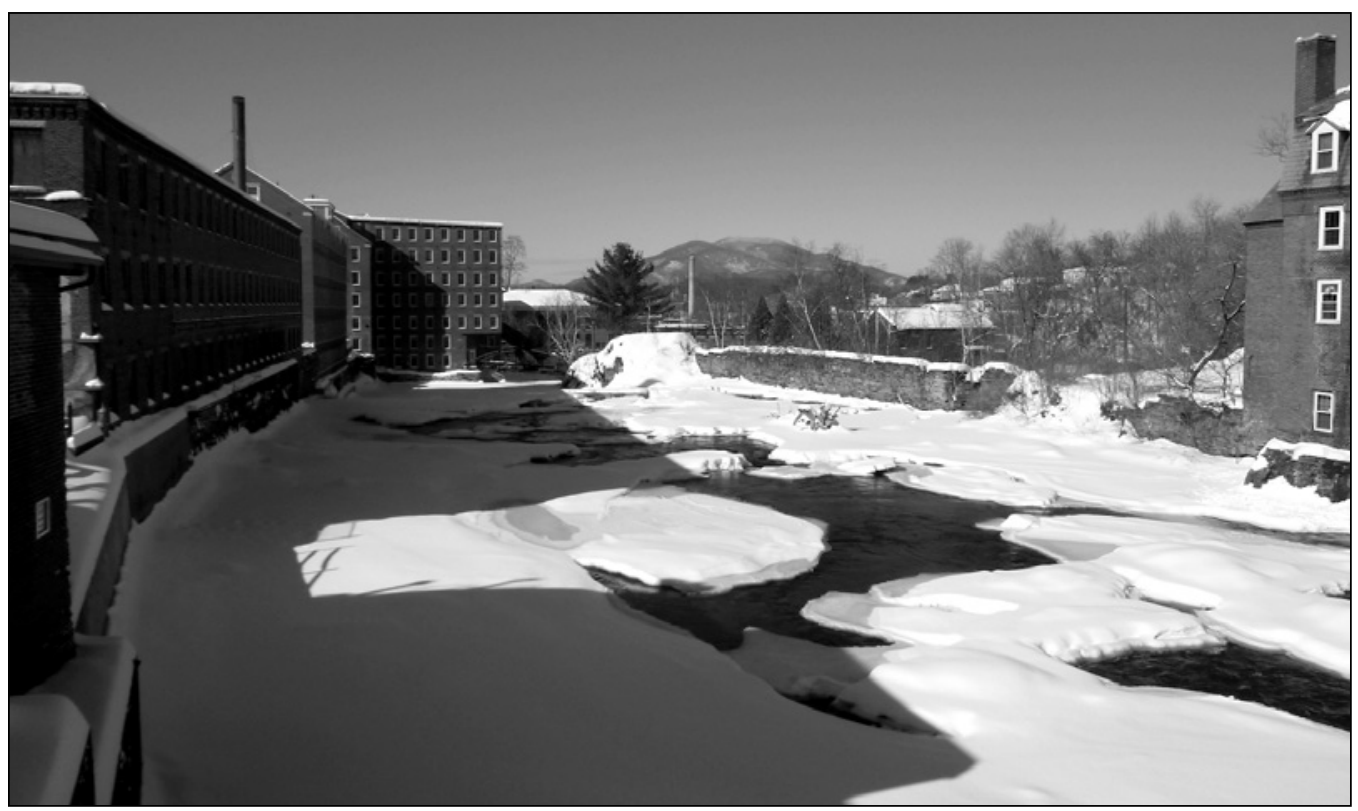

lac Champlain, cette ville de près de 40000 habitants a été à la fois un centre manufacturier important et un port de transit de marchandises. Ce riche passé a laissé bon nombre de constructions non seulement industrielles, mais aussi des édifices publics et des maisons privées qui enrichissent l'architecture de la ville. La vocation balnéaire et l'éducation, notamment avec la présence de l'Université du Vermont, sont toujours existantes à Burlington, et la ville s'est résolument tournée vers le service, laissant plutôt le secteur industriel à ses voisines. C'est ainsi que dès 1981 était inauguré le Church Street Marketplace, une zone piétonne et commerçante au centre-ville (voir illustration 3). Là encore, on remarque l'importance, au-delà de la reconnaissance patrimoniale, de la certification d'une exceptionnelle qualité de vie. Et la ville semble collectionner les titres en ce domaine : l'un des cinq lieux «où vivre et prendre sa retraite» aux États-Unis et l'un des quatre «lieux à voir» pour le magazine AARP en 2007, ou encore seulement pour 2010 le prix du Sustainable Communities Challenge délivré par le U.S. Housing and Urban Development (HUD), le prix d'excellence en développement durable du second concours de la Fondation Home Dépôt et la nomination par le magazine Kiplinger's Personal Finance dans la liste des 10 meilleurs endroits pour lancer une entreprise ou pour trouver un emploi dans la prochaine décennie. Ce type de palmarès, qui peut faire envie à de nombreuses métropoles plus importantes, semble dans ce cas prendre une importance particulière.

On le voit donc, dans ces anciennes villes industrielles, le confort des habitants est devenu une priorité. Bien sûr, il s'agit de produire une image dynamique et attractive pour rester compétitive sur le marché de la création d'entreprise, et attirer à soi de nouvelles activités économiques génératrices de développement, mais c'est aussi, sans doute, pour les habitants eux-mêmes qu'il est crucial de promouvoir ces aspects. En effet, au-delà de la création d'emploi associée aux nouvelles entreprises, les communautés des anciennes villes industrielles souffrent souvent socialement de la disparition des usines. On l'a vu dans bien des cas, encore récemment avec les fermetures de manufactures liées à l'industrie du bois ou de l'automobile, mais aussi et depuis plus longtemps dans les régions minières et sidérurgiques comme dans le Nord-Est français ou en Belgique wallonne par exemple. Dans ces contextes, la sauvegarde du patrimoine a parfois plus été un problème qu'une solution, certains trouvant inapproprié de conserver les traces d'une activité perdue et de la cause de situations souvent humainement difficiles. La seule conservation du patrimoine architectural industriel apparaît donc insuffisante si elle ne s'accompagne pas d'une "restauration» de l'image que la communauté a d'elle-même et de la fierté locale d'un passé dont les traces sont encore visibles. C'est l'une des forces de la mise en tourisme que de valoriser un objet qui devient susceptible d'intérêt pour des personnes extérieures mais aussi pour la communauté elle-même.

Il apparaît donc qu'il y a une triade d'éléments complémentaires - patrimoine, tourisme et développement durable - qui fonctionnent pour la revitalisation des villes et des communautés locales. D'abord le patrimoine, par ses valeurs propres, souligne l'originalité d'un lieu, d'une pratique et d'une communauté qui peut donc s'identifier à lui. La reconnaissance comme "rivière patrimoniale» ou de la route qui longe la Connecticut procure à chaque communauté un motif de fierté et un repère pour son identité propre. C'est aussi un élément structurant de l'action économique et du développement à travers la création d'alliance entre les différentes villes concernées et d'organismes qui promeuvent ces sites. La mise en commun de ressources permet alors de supporter mieux les charges associées à la conservation des édifices et des milieux naturels. Au patrimoine s'associe souvent le tourisme. Si son volume peut varier considérablement d'un site à l'autre, le processus de valorisation touristique, lui, ne change pas. Or 


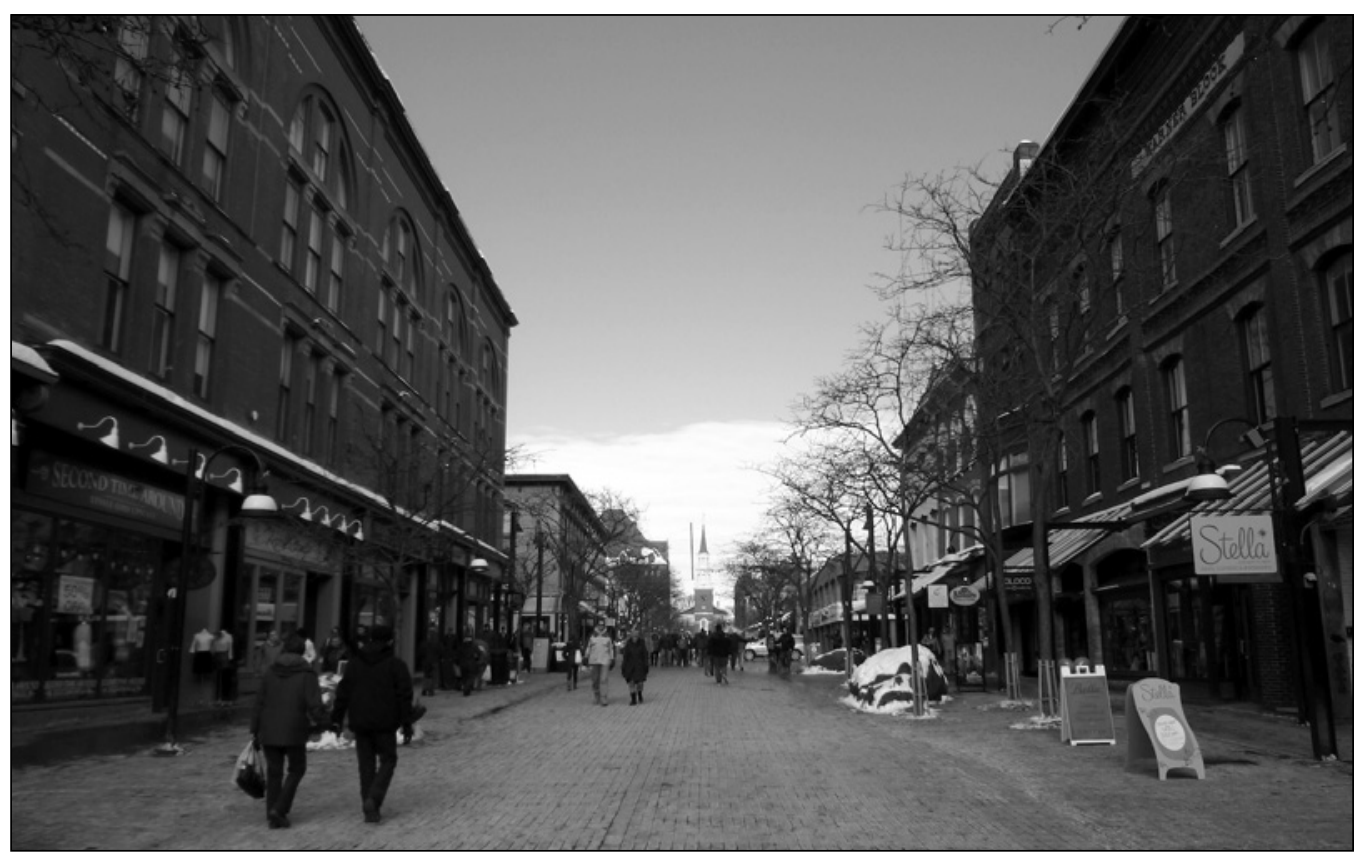

ILLUSTRATION 3 : Church Street Marketplace, Burlington, Vermont (photo : Mathieu Dormaels). bien souvent, cette valorisation se base sur les valeurs du patrimoine culturel ou naturel, insistant sur l'exceptionnalité ou la typicité, pour le rendre attractif. Ce processus de construction de l'attraction touristique, théorisé notamment par Morisset, contribue à rendre plus distinctif l'élément patrimonial, et plus évidente son importance dans la définition de l'identité de la communauté. Cependant, un troisième élément s'est adjoint à ces deux premiers.

Il n'est plus beaucoup de développements aujourd'hui qui ne doivent être durables, et le patrimoine et le tourisme n'échappent pas à ce mouvement. Dans ce cas, il s'y exprime de façon particulière. D'abord, il s'agit d'une nécessité pour la protection des biens patrimoniaux lorsque ceux-ci peuvent être victimes de détériorations majeures liées à la surfréquentation, notamment touristique. La notion de durabilité s'est donc imposée dans la gestion du tourisme patrimonial, du côté des gestionnaires de sites comme de l'industrie touristique. Toutefois, c'est aussi dans la participation de la communauté que l'on retrouve cette notion. En effet, depuis longtemps les organismes internationaux défendent l'idée qu'il faut que la communauté soit impliquée dans la préservation du patrimoine et qu'elle bénéficie des omniprésentes, quoique incalculables, retombées économiques. Il semble donc que l'on prenne de plus en plus en compte cette «qualité de vie» qui dépasse le seul confort économique pour inclure la qualité de l'environnement, le niveau de services publics ou encore la vie culturelle d'une ville ou d'un quartier. Ce faisant, la durabilité permet de consolider une image positive et valorisante pour une communauté, et de compléter une politique de développement, y compris lorsqu'elle se base sur le patrimoine et le tourisme. Les cas sont nombreux où la négligence à l'égard de la communauté locale et sa qualité de vie ont conduit à des résultats contreproductifs. Peut-être pourra-t-on d'ailleurs en présenter certains dans de futures chroniques.
Il reste que, pour ces villes au passé industriel, le patrimoine bâti est bien souvent le socle tangible d'une nouvelle dynamique locale de croissance. Toutefois, il semble évident que sa seule conservation, sans l'accompagnement d'activités nouvelles, et sans un réel effort d'intégration de la communauté et de prise en compte de sa fierté, ne saurait être viable. De nombreuses villes l'ont compris, mais pas toutes, et même ainsi, il ne peut s'agir d'un succès garanti. Pour la ville de Claremont et le reste de la vallée du Connecticut, cela reste un défi de s'assurer que les développements correspondent aux besoins locaux et permettront de promouvoir la croissance économique et sociale d'une région qu'il faut déjà prendre le temps de visiter.

\section{Notes}

1 Voir à ce sujet le numéro 21-2 (été 2002) «Patrimoine du XXe siècle» de Téoros.

\section{Références}

Connecticut river byway Council (2010) From mills to main streets, Charlestown : CRBC. 5 p.

EDMOND, Charles John (1991) From Farm to Factory. Travail d'étude du Keene State College. Non publié.

MANGIN, Claude (2002) «Géographies d'une ville industrielle américaine Lowell en modèles chronographiques ", Mappemonde, $\mathrm{n}^{\circ} 68$ (2002.4), p. 25-29.

New Hampshire Employment Security (2010) Claremont, <http://www. nh.gov/nhes/elmi/htmlprofiles/pdfs/claremont.pdf $>$, consulté le 6 février 2011.

United States Department of Transportation, Federal Highway Administration (s. d.) America’s Byways : National Scenic byways online, <http://www. byways.org/learn/program.html>, consulté le 6 février 2011.

United States Environmental Protection Agency (2010) American Heritage Rivers, <http://water.epa.gov/type/watersheds/named/heritage/>, consulté le 6 février 2011. 